\title{
Radically Open Dialectical Behavior Therapy: Social Signaling, Transdiagnostic Utility and Current Evidence
}

This article was published in the following Dove Press journal: Psychology Research and Behavior Management

\section{Kirsten Gilbert $\mathbb{D}^{1}$ \\ Karyn Hall $\mathbb{D}^{2}$ \\ R Trent Codd ${ }^{3}$}

'Department of Psychiatry, Washington University in St. Louis, St. Louis, MO, USA; ${ }^{2}$ Dialectical Behavior Therapies Center, Houston, TX, USA; ${ }^{3}$ CognitiveBehavioral Therapy Center of Western North Carolina, Asheville, NC, USA
Correspondence: Kirsten Gilbert Department of Psychiatry, Washington University in St. Louis, 4444 Forest Park, Suite 2100, St. Louis, MO 63108, USA

Tel + | 3|4-747-000|

Fax + 314-286-2732

Email gilbertk@wustl.edu

\begin{abstract}
At the core of an overcontrolled personality and coping style is a tendency to have too much self-control, exhibiting as behavioral and cognitive inflexibility, high inhibition of emotion, high detail-focused processing and perfectionism, and a lack of social connectedness. Overcontrol underlies a wide variety of psychiatric illnesses and as such, an innovative transdiagnostic therapy called Radically Open Dialectical Behavior Therapy (RO DBT) has been developed to treat disorders characterized by overcontrol. RO DBT targets maladaptive social signaling in order to help individuals "rejoin the tribe," hypothesizing that increasing social connectedness by means of targeting social signaling is the central mechanism of change in treatment. Because RO DBT is used for individuals with an overcontrolled personality style, rather than individual disordered symptoms, it can be used transdiagnostically across a range of comorbid disorders, including treatment-resistant depression and anxiety, anorexia nervosa, and personality disorders such as obsessivecompulsive personality disorder. The current article introduces this novel treatment approach and discusses its emphasis on social signaling and its transdiagnostic nature. We then provide the first review of existing literature testing the efficacy of RO DBT across clinical populations, discuss issues related to assessment of overcontrol, and speculate on future directions for this novel therapy.
\end{abstract}

Keywords: radically open dialectical behavior therapy, overcontrol, transdiagnostic, psychological inflexibility

Emotional well-being is often presumed to be positively correlated with self-control and research generally supports this presumption. ${ }^{1,2}$ Consequently, high degrees of self-control are commonly held in high esteem. However, extremes of anything are often detrimental, and there is a small but growing literature describing a non-linear relationship between self-control and emotional health, one in which the extremes at either end are associated with various forms of dysfunction. ${ }^{3-5}$ Too much selfcontrol, or an "overcontrolled" pattern of coping, is characterized by a constellation of characteristics: inflexible behavioral repertoires, emotional suppression and inhibition, elevated distress tolerance, tendencies towards perfectionism, low reward responding and high threat responding, anxious apprehension, and high detail focused processing. ${ }^{3}$ Overcontrolled coping is problematic because it is associated with a range of dysfunction, including social isolation, inferior outcomes in anxiety disorder treatment, ${ }^{6}$ and severe and difficult-to-treat mental health problems, such as anorexia nervosa, treatment-resistant depression and personality 
disorders. ${ }^{7-11}$ Because the most prevalent societal view is that greater self-control leads to better health outcomes, high self-control can be heuristically overlooked in psychological interventions or not directly targeted in therapy. Fortunately, a novel psychotherapy that directly targets overcontrolled coping, Radically Open Dialectical Behavior Therapy (RO DBT $)^{3}$ has been developed.

\section{Radically Open Dialectical Behavior Therapy (RO DBT)}

Radically Open Dialectical Behavior Therapy (RO DBT) is an evidence-based psychosocial therapy that emerged from "standard" Dialectical Behavior Therapy (DBT"M). ${ }^{12}$ DBT is a widely studied treatment originally developed for chronic suicidal behavior, subsequently was tested in borderline personality disorder, and has since been extended across disorders including substance use disorders ${ }^{13}$ and eating disorders. ${ }^{14}$ DBT is based on behaviorism, dialectical philosophy and Zen Buddhist practice and dialectically balances acceptance and change. It consists of weekly individual therapy sessions, weekly skills group sessions, as needed telephone coaching and clinician team consultation meetings. DBT transdiagnostically treats disorders characterized by high impulsivity and emotion dysregulation, or maladaptive "undercontrol," (for further discussion of undercontrol, see below).

RO DBT is also a transdiagnostic therapy; however, it treats disorders at the opposite end of the spectrum. Rather than targeting disorders of undercontrol, RO DBT targets disorders characterized by maladaptive overcontrolled coping. ${ }^{3}$ Similar to DBT, the intervention is based on behaviorism and dialectical philosophy, the intervention is 30 weeks in duration and entails concurrent weekly individual therapy and a two-and-a-half-hour skills class, with options of telephone coaching and clinician team consultation meetings.

Although a similar framework as DBT, RO DBT differs from DBT in key ways. First, the content of RO DBT individual therapy and skills class sessions differs drastically from DBT. RO DBT addresses overarching classes of behavior, or behavioral themes, that help guide treatment and identify targets related to underlying tendencies of overcontrol, including 1) hyper detail-focused and overly cautious behavior, 2) rigid and rule-governed behavior, 3 ) inhibited and disingenuous emotional expression, 4) distant and aloof social connectedness and relationships and 5) increased tendencies to engage in social comparisons, envy and bitterness. ${ }^{3}$ Second, the core problem addressed in DBT treatment is emotional dysregulation and mood-dependent behavior, while RO DBT targets emotional loneliness resulting from low openness and maladaptive social signaling. Third, and related, the mechanism of change in DBT is via targeting maladaptive action tendencies that stem from dysregulated emotions, while for RO DBT it is via targeting social signaling deficits. Fourth, DBT comes from a Zen Buddhist perspective, while RO DBT incorporates practices of Malamati Sufism. Fifth, the role of the therapist differs. In DBT the therapist is focused on helping the client not act on impulses and to be more organized while in RO DBT, the therapist is less directive and teaches the client how to be more relaxed, spontaneous, and express emotions in a more vulnerable and open way. Lastly, in RO DBT there is an emphasis on self-enquiry and self-discovery and a leaning in toward uncomfortable emotions for the purpose of learning, while in DBT the emphasis is more on external contingencies and finding ways to tolerate or regulate difficult emotions.

Training to become an RO DBT therapist includes two options. One avenue is to attend an "intensive" training that includes two separate one-week in-person trainings that are separated by six months. A second avenue is by completing a "blended learning" program that includes online webinars and shortened in-person trainings. Although these trainings provide a baseline of competency in clinically administering RO DBT, ongoing supervision of videotaped client sessions from approved RO DBT supervisors, and peer-supervision in team consultation is warranted to become proficient and adherent in effectively delivering the treatment. Certification in RO DBT is currently being developed, and self-assessment for adherence to the treatment is available in the textbook. ${ }^{3}$

\section{The Importance and Novelty of Targeting Social Signaling}

RO DBT encompasses several novel and distinctive features. For example, it uniquely challenges the heuristic of linearity with respect to self-control and healthy functioning that basic research and society often promote. ${ }^{15}$ Second and importantly, RO DBT de-emphasizes the common tendency to "go inside" the individual to alter internal states (ie, cognition, emotion) that mainstream cognitive behavioral therapy $(\mathrm{CBT})^{16}$ or DBT target. Instead, it stresses the importance of targeting social communication 
and psychological connectedness with important others, similar to interpersonal psychotherapy (IPT). ${ }^{17}$ However, RO DBT focuses on the social connectedness of a specific group of clients, those assessed as having disorders related to maladaptive overcontrol, with interventions that target specific types of social deficits.

Research has repeatedly demonstrated that a lack of physical and psychological social connection is associated with worse mental health outcomes, especially depression, ${ }^{18}$ as well as worse physical health outcomes, including mortality. ${ }^{19}$ Moreover, there is emerging research suggesting social connectedness predicts subsequent mental health (rather than vice versa) ${ }^{20}$ and an increasing body of evidence suggests that social isolation is causally implicated in the development and maintenance of depression, ${ }^{21}$ supporting the reasoning behind targeting social connectedness that some therapies, including IPT, and now RO DBT, promote.

What truly makes RO DBT unique is that RO DBT builds on this compelling literature by being the first treatment to emphasize social signaling as a primary mechanism of change. It underscores the importance of targeting publicly observable behaviors (eg, social signals) to increase psychological connectedness with important others. A social signal is defined as any action or overt behavior, regardless of its form, intent, or the performer's awareness, that is carried out in the presence of another person. ${ }^{3}$ A social signal is a form of communication, such as an eye roll, the silent treatment, walking away, or a smile. RO DBT hypothesizes that the mechanism of change in treatment is that open expression of emotion leads to trust, which leads to social connection. Mechanisms of behavior change generally refer to the underlying basic psychological processes that drive change in therapy, and for RO DBT, the theorized critical process driving change in treatment is social signaling. As such, RO DBT aims to teach overcontrolled individuals how to modify their social signaling so as to openly express emotions and vulnerabilities, which results in increased social connectedness.

RO DBT is informed by translational research, including that of evolutionary science. In order to survive, our species had to develop a way to form close bonds, share resources and work closely with genetically unrelated individuals. A quick way of communicating our intentions to others was necessary. RO DBT contends that social signals evolved to facilitate social bonds and connections and this was a key to our survival. A highly sophisticated system of social signaling allowed individuals to signal intentions or feelings through facial expressions, gestures or words. ${ }^{3}$ What we also know is that others perceive maladaptive social signaling, such as incongruent emotional expression or suppressed emotional expression, as untrustworthy or inauthentic, which in turn impairs the development of social closeness. ${ }^{22}$ It is not just important to signal openness, but also to not signal in an inauthentic manner by masking behaviors. Individuals often respond to social signals in a visceral way that may not even be in their awareness. A flat face showing no expression of emotion, for example, may create discomfort in others and a wish to not engage. Indeed, RO DBT directly targets maladaptive social signals, including micro-expressions that interfere with connecting with others and the absence of adaptive social signals.

In addition to targeting client maladaptive social signals, an additional aspect of social signaling addressed in RO DBT is how the client's maladaptive social signaling is likely to affect the therapeutic relationship. The therapy includes strategies for the therapist to help form a relationship with an individual with an overcontrolled temperament and maladaptive social signaling. This allows the client to experience an interpersonal connection and addresses the common problem of clients abandoning therapy when experiencing discomfort, conflict or perceived lack of treatment relevance. The therapist acts as a "tribal ambassador," someone who recognizes the client's lack of knowledge about how to form intimate relationships and warmly welcomes them back to the tribe.

\section{Transdiagnostic Utility}

Another distinctive feature of RO DBT is its transdiagnostic utility. Transdiagnostic treatments allow the targeting of overlapping maladaptive characteristics across comorbid psychiatric conditions. This is desirable because comorbidity is common and substantial in mental health ${ }^{23}$ and the number of treatments for individual disorders is high, requiring significant time and money from clinicians to achieve and maintain competency in multiple protocols. ${ }^{24}$ Contemporary examples of transdiagnostic therapies include the unified protocol for transdiagnostic treatment for emotional disorders (UP) designed for anxiety and mood disorders, Acceptance Commitment Therapy (ACT) ${ }^{25}$ developed to target any disorder in which experiential avoidance is a core feature and DBT ${ }^{\mathrm{TM}},{ }^{12}$ utilized for disorders of undercontrol, including borderline personality disorder. 
RO DBT extends the transdiagnostic tradition by addressing unique treatment targets in subsets of individuals with differing symptom presentations but a similar underlying temperamental and coping style. Specifically, RO DBT targets maladaptive overcontrol across treatmentresistant mood and anxiety disorders (eg, depression, social phobia, generalized anxiety disorder and obsessive compulsive disorder), eating disorders (anorexia nervosa) and personality pathology (eg, obsessive compulsive personality disorder, avoidant personality disorder). Of note, although mood and anxiety disorders often respond favorably to first-line interventions such as cognitive behavioral approaches, those individuals that do not respond to effective first-line treatments may experience treatment resistance because of underlying personality tendencies and overlearned perceptual and regulatory biases. ${ }^{26}$ As such, when first-line treatments such as cognitive behavior therapy ${ }^{16}$ are ineffective, assessing for underlying tendencies of overcontrol may provide direction. If maladaptive overcontrol is evident, RO DBT is indicated. Where RO DBT may be a first-line treatment is specifically for personality disorders, including obsessive compulsive personality disorder and related Cluster $\mathrm{A}$ and $\mathrm{C}$ disorders that are characterized by overcontrol as well as for anorexia nervosa (while also ensuring that a separate team is helping manage weight restoration and medical issues). There are no currently empirically proven superior treatments for anorexia $^{27}$ and RO DBT has effectively been tested (demonstrating large effect sizes, see below) in anorexia in both inpatient and outpatient settings. ${ }^{28,29}$

Taken together, anxiety, mood and eating disorders demonstrate high comorbidities with each other as well as with personality disorders. ${ }^{9,30-32}$ As such, RO DBT purports that "personality matters" in the treatment of many psychological disorders and thus targets underlying personality components and tendencies that occur across disorders. ${ }^{3}$ For those individuals who do not respond to first-line treatments and whose psychiatric illness is characterized by maladaptive overcontol, RO DBT may be warranted. The growing empirical literature on RO DBT supports the importance of intervening with this personality and coping style.

\section{Current Evidence Base}

Although RO DBT is still just beginning to be widely disseminated, there is already a foundation supporting its efficacy as shown by the following review of all peerreviewed published studies that have clinically tested RO
DBT. The most robust evidence of the efficacy of RO DBT is from a multi-site randomized controlled trial (RCT) that tested RO DBT versus treatment as usual (TAU) for adult patients with treatment refractory depression. In order to be included in the study, participants needed to be currently depressed, have experienced at least two previous depressive episodes or chronic depression, and, must have been non-responsive to at least 6 weeks of antidepressant medication..$^{33}$ Moreover, $96 \%$ of participants met the criteria for at least one comorbid disorder $(86 \%$ at least one DSM-IV Axis I disorder and 78\% for at least one DSM-IV Axis II disorder). ${ }^{33}$ As anticipated, participants were difficult-to-treat non-responders to other pharmacologic and therapeutic intervention with high comorbidity and heterogeneity. Findings from this RCT indicated that participants in RO DBT $(\mathrm{n}=162)$ experienced significantly reduced depressive symptoms compared with TAU following treatment $(\mathrm{n}=88 ; d=1.03) .{ }^{26}$ However, this significant difference did not survive significance testing at 4-month follow-up, although still trended in the same direction. Full remission rates were low across both treatments; however, partial remission rates were non-significantly higher in RO DBT (23\% post-treatment at 7 months, $26 \%$ at 12 months and $33 \%$ at 18 months) compared with TAU $(6 \%, 22 \%$ and $24 \%$ at 7,12 , and 18 months, respectively), as were participants' subjective report of reliable improvement and worthwhile change. Additionally, participants in RO DBT demonstrated significant increases in emotional coping and decreases in psychological inflexibility. Limitations of this multi-site RCT include a smaller than planned sample size great and therapist heterogeneity in patient outcomes ${ }^{26}$ possibly indicating difficulty delivering the treatment. Despite these limitations, the first multi-site RCT of RO DBT was effective in decreasing depressive symptoms immediately posttreatment (with a large effect size) as well as improving psychological functioning related to overcontrolled tendencies (eg, emotional coping and flexibility) over followup.

Earlier pilot studies on RO DBT were completed in samples of older adults with non-responding chronic depression. ${ }^{34}$ In an initial pilot study, RO DBT demonstrated equivalent decreases in depression post-therapy $(\mathrm{n}=16, d=2.51)$ when compared with standard medication treatment $(\mathrm{n}=15 ; d=2.24) .{ }^{35}$ However, outcomes differed at the six month follow-up: participants exposed to RO DBT exhibited significantly fewer depressive symptoms compared with medication treatment $(d=0.98) .{ }^{35}$ In 
a separate study and the first RCT comparing medication alone and RO DBT plus medication in chronically depressed older adults $(n=34)$, groups demonstrated nonsignificantly different levels of remission post-treatment ( $\mathrm{n}=34 ; d=0.14$ and $d=0.39$ for the Hamilton Rating Scale of Depression ${ }^{36}$ and Beck Depression Inventory, ${ }^{37}$ respectively). ${ }^{38}$ However, again, at six month follow up, RO DBT showed superior outcomes with significantly higher remission rates compared with medication alone (unable to calculate effect size). ${ }^{38}$ In a second RCT that included chronically depressed older adults with comorbid personality disorders who first underwent 8 weeks medication trial prior to either continuing with TAU medication or adding RO DBT to ongoing medication $(n=37)$, there were no differences in the two groups following skills group completion $(d=0.49)$, following individual treatment $(d=0.34)$, or at a six-month follow-up $(d=0.30)$. However, compared with medication only, RO DBT plus medication led to significant decreases in interpersonal sensitivity and interpersonal aggression, both of which are characteristic relational problems that typify personality disorders. ${ }^{38}$ Of note, limitations of both above studies include small sample size, increased time spent with patients in the RO DBT condition, and an earlier version of RO DBT than is used in standard practice currently. Together, initial evidence using an earlier version of the treatment demonstrated no significant differences from ongoing medication management immediately post-treatment, but preliminary indication that the longer term effects of RO DBT compared with medication are enhanced in coping and relational outcomes.

RO DBT has also been evaluated in anorexia nervosa. No one treatment has demonstrated superior efficacy in the treatment of anorexia ${ }^{27}$ and because anorexia is characterized by poor flexibility and social connectedness and high emotional inhibition and threat sensitivity, it has been conceptualized as a prototypical disorder of overcontrol. ${ }^{7}$ In an initial feasibility study, RO DBT was implemented in an inpatient setting for anorexia $(n=47)$ and demonstrated a high completion rate of treatment $(72 \%){ }^{28}$ For those treatment completers, RO DBT led to a significant increase in BMI $(d=1.91)$. RO DBT also led to significant improvements in eating disordered symptoms $(d=1.17)$, quality of life $(d=0.1 .03)$, and significant reductions in distress $(d=1.34)$. However, post-treatment follow-up data were not obtained. In a second pilot study, patients with anorexia from an adult outpatient eating disorders clinic $(n=9)$ who participated in RO DBT demonstrated decreases in eating disorder behaviors and symptoms and medical problems associated with anorexia (eg, anemia, osteoporosis). ${ }^{29}$ Importantly, 55\% of the sample regained regular menses by 12-month follow up (none reported regular menses at baseline) and improvements in BMI post-treatment were maintained at six and twelve months follow up (effect size $d$ 's $=-1.12,-0.87$, and-1.12 at posttreatment, $6 \mathrm{mo}$ and $12 \mathrm{mo}$ follow-up, respectively). Moreover, RO DBT led to fewer personality and other comorbid disorders post-treatment (eg, obsessive compulsive personality disorder (OCPD), avoidant personality disorder (APD), suicide attempts, social phobia) as well as increased global functioning. ${ }^{29}$ Of note though, this second study was a case series design and a small sample size with uncontrolled treatment duration limits generalizability. Notably, although not initially designed to treat anorexia nervosa, RO DBT appears to be preliminarily effective in a notoriously treatment resistant patient population, demonstrating large effect sizes and significant improvements not only in eating disordered pathology, but also in comorbid pathology and functioning.

Rather than treating specific disorders (eg, depression or anorexia), RO DBT has also been adapted for use in clinically heterogeneous overcontrolled populations. For instance, a psychiatric hospital outpatient sample $(n=117)$ participated in RO DBT skills or treatment as usual (TAU). ${ }^{39}$ RO DBT participants demonstrated significantly greater reductions in overall psychopathology $(d=1.67)$. They also exhibited significantly greater improvements in coping skills $(d=0.81)$, perceptions of safety in social environments $(d=0.50)$, and decreases in need for structure $(d=0.41)$ compared with TAU. Moreover, at 3-month follow-up, significant improvements remained in skills use and decreased need for structure in the RO DBT group compared with TAU. Limitations include a lack of random assignment to conditions and lack of follow-up in the TAU group. This initial finding demonstrates medium to large effect sizes and preliminary utility in heterogeneous samples characterized by an overcontrolled personality.

\section{Assessment of Overcontrol in Psychiatric Illness}

Overcontrol in itself is not a diagnosable psychiatric illness, nor is it always maladaptive, and as such, it is imperative to take care in assessing overcontrol in treatment-seeking populations to consider whether RO DBT is a good fit. Specifically, maladaptive overcontrol is 
a multifaceted phenotype that emerges from complex transactions among biology, environment, and individual styles of coping. ${ }^{3}$ It is characterized by low receptivity and openness, high rigidity or inflexibility, high detail focused processing, pervasive inhibited or suppressed emotional expression, rule-governed behaviors and strong moral convictions, and low social connectedness. Although features of overcontrol share much in common with symptoms of OCPD, overcontrol is conceptually different and should be distinguished from this personality disorder as part of assessment. First, overcontrol is a multifaceted dimensional phenotype that is not always maladaptive, but rather, in some instances is related to success and can be evident in high functioning individuals. ${ }^{3}$ Second, although the diagnostic criteria for OCPD are prototypical examples of maladaptive overcontrolled coping, OCPD does not necessarily include the full spectrum of how maladaptive overcontrol can evidence. Indeed, RO DBT posits that an overcontrolled presentation can present as one of two lower-order subtypes: overly disagreeable and overly agreeable. ${ }^{3}$ Although much of the thinking on these subtypes is still speculative, overly disagreeable overcontrolled individuals tend to value fairness, being correct, and appearing competent above all else, ${ }^{3}$ and this subtype is theoretically more closely associated with OCPD. Overly agreeable overcontrolled individuals value maintaining the peace and appearing liked by others, and this subtype is theoretically more closely associated with avoidant personality disorder. Assessing for these subtypes is important in order to tailor RO DBT to the specific presentation of overcontrol (see ${ }^{3}$ for assessment of subtypes).

Another quick and initial strategy to assess overcontrol is through the use of questionnaires. Although the development of validated assessments of overcontrol is ongoing, there are available self-reported questionnaires for adults, $^{3}$ adolescents ${ }^{40}$ and parents about their children $^{41}$ that can be used as an initial assessment. In adults, the Brief Overcontrol Scale demonstrates norms of dimensional overcontrol among males and females and ongoing work is testing clinical cut-offs. ${ }^{42}$ For adolescent populations, a screener differentiating maladaptive overcontrol and undercontrol traits in an adolescent community population is currently undergoing validation (the Over and Undercontrolled Traits Measure for Adolescents; OUT-Ma $)^{40}$. Lastly, to identify early manifestations of overcontrol when it is first thought to become stable (age $\sim 5-6$ years) ${ }^{43}$ the Overcontrol in Youth Checklist (OCYC) was developed. ${ }^{41}$ The OCYC is a parent-reported assessment that demonstrates associations with elevated child behavioral inhibition, cognitive deficits, internalizing and externalizing symptoms and social functioning deficits, as well as neural aberrations in error monitoring. ${ }^{41,44}$ It will be imperative for future work to not only assess dimensional overcontrol, but also to create standardized clinical cut-offs that identify when overcontrol becomes pathological.

In addition to self or parent-reported measures of overcontrol, a clinical interview is essential. This is of particular importance when distinguishing between maladaptive overcontrol and undercontrol. ${ }^{45}$ Undercontrol is characterized by low inhibitory control, global focused processing, high reward sensitivity and chaotic relationships. Borderline personality disorder is a prototypical disorder of undercontrol. Assessing social signaling is warranted in distinguishing over- and undercontrolled individuals: overcontrolled individuals are inhibited, controlled, and mood independent while undercontrolled individuals tend to be dramatic, disinhibited and mood-driven in their social signaling. ${ }^{45}$ Another critical and unique factor to consider in assessment is the context of behavior: whether a behavior occurs in public or in private. A public setting means that the behavior occurs in the presence of another person who is not in the immediate family or similar social analogue while a private setting is any situation in which a person believes he is unidentifiable, anonymous, or alone (or with very close others). While individuals with overcontrolled temperament typically inhibit displays of emotion, especially in public settings, they may show emotional leakage (dramatic displays of emotions such as yelling, rage, tearfulness and other expressions of intense emotions) in private settings.

Indeed, a failure to distinguish between public and private behavior is believed to be one of the causes of mislabeling overcontrol as undercontrol. ${ }^{3}$ Specifically, overcontrolled individuals may endorse symptoms of undercontrolled diagnoses in standard clinical assessments. This was demonstrated in a RCT of overcontrolled adults with treatment-resistant depression, ${ }^{26,46}$ during which overcontrolled clients endorsed up to nine symptoms of borderline personality disorder, a prototypical disorder of undercontrol. ${ }^{45}$ After case consultation, many individuals endorsing symptoms were deemed overcontrolled, suggesting that a number of symptoms that appear to reflect undercontrolled tendencies (ie, self-harm/suicidality, chronic emptiness, identity disturbance), can also be 
problems reported by individuals with overcontrol. As such, it may be important for clinicians to ask more specific details about how the symptoms are expressed (ie, socially signaled), in what context, and to what degree the behavior is expressed (eg, a stern tone versus breaking things when in a rage).

\section{Future Directions and Questions}

Overcontrol is rooted in personality style, and as such, develops early and underlies multiple forms of psychopathology. The beneficial outcomes of targeting overcontrol in RO DBT have only begun to be tapped, as RO DBT could have farreaching effects across various presentations. To start, overcontrol purportedly becomes stable around age five ${ }^{43}$ and remains generally stable across the lifespan. ${ }^{47}$ As such, identifying maladaptive overcontrol in young children is imperative to begin intervening earlier. ${ }^{41}$ Similarly, overcontrol in childhood is associated with many of the same outcomes as adults: social functioning problems such as withdrawal and loneliness as well as internalizing and anxiety symptoms. ${ }^{41,48,49}$ Developmentally adapting RO DBT to young children and their caregivers may provide a window of opportunity to make a large impact, by targeting overcontrolled coping styles when they are more malleable for change and helping teach skills that could have lifelong impact.

Related to targeting young children, research has demonstrated that working with families of those diagnosed with mental disorders leads to better outcomes. ${ }^{50,51}$ As such, research is needed to determine if and how working with families of maladaptively overcontrolled individuals (both as children and adults) leads to beneficial outcomes such as improved prognosis of the OC individual. The biosocial theory for overcontrol specifics factors that give rise to maladaptive $\mathrm{OC}$, including environments that provide reinforcing contingencies for perfectionism, inhibiting emotions, winning, following rules, and tolerating pain. Punishing contingencies for mistakes, showing vulnerability and requesting nurturance may also be present. Teaching family members skills and ways of responding that do not reinforce overcontrolled responding would seem to be an effective intervention, but that research remains to be done. Addressing maladaptive overcontrol and undercontrol in couples' work brings additional considerations, such as how to most effectively intervene with different combinations of undercontrolled and overcontrolled couples.

The use of RO DBT with forensic populations is also a potentially promising area for future investigation.
Although antisocial behavior is commonly thought to be characterized by an undercontrolled personality style because of poor impulse control, as early as the 1960s a subtype of overcontrolled violent offender was identified. $^{52,53}$ However, there have been limited highquality studies examining psychological interventions for this purported overcontrolled offender subtype. Because RO DBT was designed to specifically target overcontrolled coping, a line of research has been initiated with this violent offender subtype. ${ }^{54}$ Preliminary findings confirmed several predictions derived from the RO DBT model. Specifically, the overcontrolled sub-type was prevalent (44\%) and hypothesized bio-temperamental biases were observed in a sample of offenders. ${ }^{54}$ In addition, relative to undercontrolled offenders, overcontrolled offenders were less socially connected to others (ie, social detachment and independent staff ratings). To this end, a trial of RO DBT with violent offenders in a maximum-security setting is currently underway.

Another novel avenue for future research in RO DBT is in individuals adjusting to chronic illness. Research has repeatedly demonstrated a bi-directional relationship between mental and physical health disorders ${ }^{55}$ and other psychological treatments, including Acceptance and Commitment Therapy (ACT), ${ }^{25}$ have demonstrated efficacy in reducing psychological symptoms and improving symptom management in patients with HIV, chronic pain and cystic fibrosis. ${ }^{56}$ However, many individuals remain symptomatic after undergoing the best available interventions, such as ACT, and one could speculate that RO DBT's focus on targeting personality could improve outcomes for these treatment non-responders characterized by overcontrol. For example, overcontrolled coping, including rigidity and rejection of help, could exacerbate chronic health conditions. Additionally, solid organ transplantation guidelines require the presence of a solid care plan, implying that patients who are socially isolated or unable to form close relationships possibly resulting from specific personality tendencies are at a decided disadvantage. Indeed, there is an ongoing pilot study examining RO DBT for overcontrolled patients living with chronic health conditions. $^{57}$

In summary, personality seems to matter when treating chronic, comorbid and difficult to treat disorders. RO DBT provides a novel treatment approach that directly targets social connectedness via a unique mechanism of change: social signaling. Although initial studies testing RO DBT in chronically depressed and treatment-resistant samples indicated no added benefits of RO DBT to ongoing 
medication treatment immediately post-treatment, these studies hinted at longer-term change in better coping and remission. Moreover, more recent studies using an updated manual and examining RO DBT in populations characterized by overcontrol, (eg, anorexia, clinically heterogeneous overcontrolled samples and treatment-resistant depression characterized by an overcontrolled style of coping) demonstrate larger effect sizes.

RO DBT is a novel and innovative treatment that can be employed for those individuals who display an overcontrolled personality and for whom first-line interventions are not effective. Initial research supports RO DBT to be an effective intervention for chronic and treatment-resistant mental illnesses. Moreover, findings from the current review also highlight the importance of accurately assessing and identifying overcontrol to ensure that RO DBT is a good fit for the client. Although a growing body of evidence supporting RO DBT is emerging, there is still much research on assessing overcontrol and testing RO DBT in various treatment-seeking samples that has yet to be done.

\section{Acknowledgment}

Funding for work on this manuscript was provided by National Institute of Mental Health to K.G. (K23MH115074).

\section{Disclosure}

Dr Karyn Hall reports personal fees from Radically Open LTD, outside the submitted work; and an approved supervisor for RO DBT. The authors report no other conflicts of interest in this work.

\section{References}

1. Moffitt TE, Arseneault L, Belsky D, et al. A gradient of childhood self-control predicts health, wealth and public safety. Proc Natl Acad Sci U S A. 2011;108:2693-2698. doi:10.1073/pnas.1010076108. PMCID: PMC3041102.

2. Wiese CW, Tay L, Duckworth AL, et al. Too much of a good thing? Exploring the inverted-U relationship between self-control and happiness. J Pers. 2018;86(3):380-396. doi:10.1111/jopy.12322

3. Lynch TR. Radically Open Dialectical Behavior Therapy: Theory and Practice for Treating Disorders of Overcontrol. Oakland (CA): Context Press; 2018.

4. Grant AM, Schwartz B. Too much of a good thing: the challenge and opportunity of the inverted U. Perspect Psychol Sci. 2011;6:61-76. doi: $10.1177 / 1745691610393523$

5. Carter NT, Guan L, Maples JL, Williamson RL, Miller JD. The downsides of extreme conscientiousness for psychological well-being: the role of obsessive compulsive tendencies. J Pers. 2016;84(4):510-522. doi:10.1111/jopy.12177. PubMed PMID: 116709152.

6. Massion AO, Dyck IR, Shea MG, Phillips KA, Wasrshaw MG, Keller MB. Personality disorders and time to remission in generalized anxiety disorder, social phobia, and panic disorder. Arch Gen Psychiatry. 2002;59(5):434-440. doi:10.1001/archpsyc.59.5.434
7. Hempel RJ, Vanderbleeck E, Lynch TR. Radically open DBT: targeting emotional loneliness in anorexia nervosa. Eat Disord. 2018;26:92-104. doi:10.1080/10640266.2018.1418268

8. Lynch TR, Hempel RJ, Clark LA. Promoting radical openness and flexible control. In: Livesley J, Dimaggio G, Clarkin J, editors. Integrated Treatment for Personality Disorder. New York: Guilford Publications; 2015:325-344.

9. Zucker NL, Losh M, Bulik CM, LaBar KS, Piven J, Pelphrey KA. Anorexia nervosa and autism spectrum disorders: guided investigation of social cognitive endophenotypes. Psychol Bull. 2007;133 (6):976-1006. doi:10.1037/0033-2909.133.6.976. PubMed PMID: 17967091.

10. Riso LP, Miyatake RK, Thase ME. The search for determinants of chronic depression: a review of six factors. $J$ Affect Disord. 2002;70 (2):103-115. doi:10.1016/S0165-0327(01)00376-7

11. Pinto A, Greene AL, Storch EA, Simpson HB. Prevalence of childhood obsessive-compulsive personality traits in adults with obsessive compulsive disorder versus obsessive compulsive personality disorder. J Obsessive Compuls Relat Disord. 2015;4:25-29. doi:10.1016/j.jocrd.2014.11.002. PubMed PMID: 25574456; PMCID: PMC4283832.

12. Linehan MM. Cognitive-Behavioral Treatment of Borderline Personality Disorder. New York (NY): Guilford Press; US; 1993.

13. Dimeff LA, Linehan MM. Dialectical behavior therapy for substance abusers. Addict Sci Clin Pract. 2008;4(2):39-47. doi:10.1151/ ascp084239. PubMed PMID: 18497717.

14. Linehan MM, Chen EY. Dialectical behavior therapy for eating disorders. In: Freeman A, editor. Encyclopedia of Cognitive Behavior Therapy. New York: Springer Press; 2005:168-171.

15. Luoma JB, Codd RT, Lynch TR. Radically open dialectical behavior therapy: shared features and differences with ACT, DBT and CFT. Behav Ther. 2018;41(3):142-149.

16. Beck A, Rush AJ, Shaw BF, Emery G. Cognitive Therapy of Depression. New York: The Guilford Press; 1979.

17. Weissman MM, Markowitz JC, Klerman GL. Comprehensive Guide to Interpersonal Psychotherapy. New York: Basic Books; 2000.

18. Donovan NJ, Wu Q, Rentz DM, Sperling RA, Marshall GA, Glymour MM. Loneliness, depression and cognitive function in older U.S. adults. Int J Geriatr Psychiatry. 2017;32(5):564-573. doi: $10.1002 /$ gps.v32.5

19. Holt-Lunstad J, Smith TB, Baker M, Harris T, Stephenson D. Loneliness and social isolation as risk factors for mortality: a meta-analytic review. Perspect Psychol Sci. 2015;10(2):227-237. doi: $10.1177 / 1745691614568352$

20. Saeri AK, Cruwys T, Barlow FK, Stronge S, Sibley CG. Social connectedness improves public mental health: investigating bidirectional relationships in the New Zealand attitudes and values survey. Aust N Z J Psychiatry. 2019;52(4):365-374. doi:10.1177/0004867417723990

21. Cruwys T, Dingle GA, Haslam C, Haslan SA, Jetten J, Morton TA. Social group memberships protect against future depression, alleviate depression symptoms and prevent depression relapse. Soc Sci Med. 2013;98:179-185. doi:10.1016/j.socscimed.2013.09.013

22. Butler EA, Egloff B, Wlhelm FH, Smith NC, Erickson EA, Gross JJ. The social consequences of expressive suppression. Emotion. 2003;3 (1):48-67. doi:10.1037/1528-3542.3.1.48. PubMed PMID: 200302341-008.

23. Plana-Ripoll O, Pedersen CB, Holtz Y, et al. Exploring comorbidity within mental disorders among a Danish national population. JAMA Psychiatry. 2019;76(3):259-270. doi:10.1001/jamapsychiatry.2018.3658

24. Gros DF, Allan NP, Szafranski DK. Movement towards transdiagnostic psychotherapeutic practices for the affective disorders. Evidenced Based Mental Health. 2016;19:e10-e12. doi:10.1136/eb-2015102286

25. Hayes SC, Strosahl KD, Wilson KG. Acceptance and Commitment Therapy: The Process and Practice of Mindful Change. New York (NY): Guilford Press; 2009. 
26. Lynch TR, Hempel RJ, Whalley B, et al. Refractory depression mechanisms and efficacy of radically open dialectical behaviour therapy (RefraMED): findings of a randomised trial on benefits and harms. Br J Psychiatry. 2019;1-9. doi:10.1192/bjp.2019.53. Epub 07/18.

27. Watson HJ, Bulik CM. Update on the treatment of anorexia nervosa: review of clinical trials, practice guidelines and emerging interventions. Psychol Med. 2013;43(12):2477-2500. doi:10.1017/ S0033291712002620. PubMed PMID: 23217606.

28. Lynch TR, Gray KL, Hempel RJ, Titley M, Chen EY, O'Mahen HA. Radically open-dialectical behavior therapy for adult anorexia nervosa: feasibility and outcomes from an inpatient program. $B M C$ Psychiatry. 2013;13:293. doi:10.1186/1471-244X-13-293. PubMed PMID: 24199611; PMCID: PMC3875355.

29. Chen EY, Segal K, Weissman J, et al. Adapting dialectical behavior therapy for outpatient adult anorexia nervosa-a pilot study. Int $J$ Eat Disord. 2015;48(1):123-132. doi:10.1002/eat.22360. PubMed PMID: 25346237.

30. Martinussen M, Friborg O, Schmierer P. The comorbidity of personality disorders in eating disorders: a meta-analysis. Eat Weight Disord. 2017;22(2):201-209. doi:10.1007/s40519-016-0345-x

31. Friborg O, Martinsen EW, Martinussen M, Kaiser S, Øvergård KT, Rosenvinge JH. Comorbidity of personality disorders in mood disorders: a meta-analytic review of 122 studies from 1988 to 2010 . J Affect Disord. 2014;152-154:1-11. doi:10.1016/j.jad.2013.08.023

32. Levinson CA, Zerwas SC, Brosof LC, et al. Associations between dimensions of anorexia nervosa and obsessive-compulsive disorder: an examination of personality and psychological factors in patients with anorexia nervosa. Eur Eating Disord Rev. 2019;27(2):161-172. doi:10.1002/erv.2635

33. Lynch TR, Whalley B, Hempel RJ, et al. Refractory depression: mechanisms and evaluation of radically open dialectical behaviour therapy (RO-DBT) [REFRAMED]: protocol for randomised trial. BMJ Open. 2015;5(7):e008857. doi:10.1136/bmjopen-2015-008857. PubMed PMID: 26187121; PMCID: PMC4513446.

34. Lynch TR. Treatment of elderly depression with personality disorder comorbidity using dialectical behavior therapy. Cogn Behav Pract. 2000;7:468-477. doi:10.1016/S1077-7229(00)80058-9

35. Lynch TR, Morse JQ, Mendelson T, Robins CJ. Dialectical behavior therapy for depressed older adults: a randomized pilot study. $\mathrm{Am}$ $J$ Geriatr Psychiatry. 2003;11(1):33-45. doi:10.1097/00019442200301000-00006

36. Hamilton M. A rating scale for depression. J Neurol Neurosurg Psychiatry. 1960;23:56-61. doi:10.1136/jnnp.23.1.56

37. Beck A, Steer RA, Brown GK. Manual for the Beck Depression Inventory-II. San Antonio (TX): Psychological Corporation; 1996.

38. Lynch TR, Cheavens JS, Cukrowicz KC, Thorp SR, Bronner L, Beyer J. Treatment of older adults with co-morbid personality disorder and depression: a dialectical behavior therapy approach. Int J Geriatr Psychiatry. 2007;22(2):131-143. doi:10.1002/gps.1703. PubMed PMID: 17096462.

39. Keogh KB, Baird K, Gibson J, Davenport J. The Radical Openness Group: a controlled trial with 3-month follow-up. Pract Innovations. 2016;1(2):129-143. doi:10.1037/pri0000023

40. James P, Stewart C, Simic M, Hempel RJ, Seretis D, Carr S. The over and under controlled traits measure for adolescents: A community validation study in preparation.
41. Gilbert KE, Barch DM, Luby J. The overcontrol in youth checklist: validation of a behavioral measure of overcontrol in preschool aged children. Child Psychiatry Hum Dev. 2019. doi:10.1007/s10578-01900907-3

42. Seretis D, Maguire T, Hempel RJ, Lynch TR, Hart C. The brief overcontrol scale [Internet]. 2017.

43. Eisenberg N, Spinrad TL, Eggum ND. Emotion-related self-regulation and its relation to children's maladjustment. Annu Rev Clin Psychol. 2010;6:495-525. doi:10.1146/annurev.clinpsy.121208.131208. PMCID: PMC3018741.

44. Gilbert K, Perino MT, Myers MJ, Sylvester CM. Overcontrol and neural response to errors in pediatric anxiety disorders under review.

45. Hempel RJ, Rushbrook SC, O’Mahen H, Lynch TR. How to differentiate overcontrol from undercontrol: findings from the RefraMED study and guidelines from clinical practice. Behav Ther. 2018;41:3.

46. Lynch TR, Hempel RJ, Whalley B, et al. Radically open dialectical behaviour therapy for refractory depression: the RefraMED RCT. Efficacy Mech Eval. 2018;5(7):1-112. doi:10.3310/eme05070

47. Asendorpf JB, Borkenau P, Ostendorf F, Van Aken MAG. Carving personality description at its joints: confirmation of three replicable personality prototypes for both children and adults. Eur J Pers. 2001;15(3):169-198. doi:10.1002/per.408

48. Eisenberg N, Spinrad TL, Morris AS. Regulation, resiliency, and quality of social functioning. Self Identity. 2002;1:121-128. doi:10.1080/152988602317319294

49. Robins RW, John OP, Caspi A, Moffitt TE, Stouthamer-Loeber M. Resilient, overcontrolled, and undercontrolled boys: three replicable personality types. J Pers Soc Psychol. 1996;70(1):157-171. doi:10.1037/0022-3514.70.1.157.

50. Dixon LB, Lehman AF. Family interventions for schizophrenia. Schizophr Bull. 1995;21(4):631-643. doi:10.1093/schbul/21.4.631

51. Falloon IRH. Family interventions for mental disorders: efficacy and effectiveness. World Psychiatry. 2003;2(1):20-28.

52. Megargee EI. Undercontrolled and overcontrolled personality types in extreme antisocial aggression. Psychol Monogr. 1966;80(3):611. doi: $10.1037 / \mathrm{h} 0093894$

53. Hamilton L, Bacon L, LongFellow E, Tennant A. Not everything is as it seems: RO DBT and overcontrolled disorders in forensic settings. Behav Ther. 2018.

54. Hamilton L, Smith-Lynch E, Hempel RJ, Lynch TR, Winder B. An examination of a new neurobiosocial theory of overcontrol with personality disordered offenders in preparation.

55. Hooten WM. Chronic pain and mental health disorders: shared neural mechanisms, epidemiology, and treatment. Mayo Clin Proc. 2016;91 (7):955-970. doi:10.1016/j.mayocp.2016.04.029

56. O'Hayer CVF, Bennett DS, Jacobson J. The lasting impact of HIV/ ADS. In: Skinta M, Curtin A, editors. Mindfulness and Acceptance for Gender \& Sexual Minorities. New Harbinger/Context Press; 2016: 227-246.

57. O'Hayer CVF, Nurse CN. Maladaptive overcontrol among people living with chronic illness: application of Radically open DBT to behavioral medicine populations in preparation. 


\section{Publish your work in this journal}

Psychology Research and Behavior Management is an international, peer-reviewed, open access journal focusing on the science of psychology and its application in behavior management to develop improved outcomes in the clinical, educational, sports and business arenas. Specific topics covered in the journal include: Neuroscience, memory and decision making; Behavior modification and management; Clinical applications; Business and sports performance management; Social and developmental studies; Animal studies. The manuscript management system is completely online and includes a very quick and fair peer-review system, which is all easy to use. Visit http://www. dovepress.com/testimonials.php to read real quotes from published authors. 